\title{
HISTORIA ORAL DE LOS CREOLES DE CORN ISLAND
}

\section{Ella Jean Downs}

La isla de Corn Island se encuentra ubicada 40 millas náuticas al Noreste del puerto El Bluff. Limita en todo su contorno con el Mar Caribe. Su extensión territorial (Great y Little Corn Island) es de $13.1 \mathrm{~km}^{2}$. Su población aproximada alcanza los 9,000 habitantes y la lengua predominante es el inglés kriol. El pueblo afro-descendiente creole de este municipio de la Región Autónoma del Atlántico Norte vive disperso alrededor de la isla. Los barrios tradicionalmente con mayor concentración de nativos creoles han sido Quinn Hill (La Loma), South End, Salli Peachi y North End.

A través de esta investigación se provee a la comunidad de los conocimientos básicos sobre el origen, las tradiciones y costumbres de nuestros ancestros. El elemento básico de este estudio lo constituye la participación de trece portadores culturales ubicados en los diferentes sectores y barrios de la comunidad de Corn Island. Cabe mencionar que desde 1985 se inició de manera informal este proceso de recopilación oral de la historia de Corn Island.

Este esfuerzo de recopilación se originó por la necesidad sistematizar la historia de la Isla según el conocimiento que han tenido de ella nuestros ancestros y para identificar los portadores culturales de esta historia que aún quedan en la Isla. En este sentido, los entrevistados escogidos para las entrevistas fueron trece, seis mujeres, entre $80 \mathrm{y}$ 90 años, y siete varones entre 65 y 90 años. Entre estos trece participantes se encuentran líderes comunales, líderes religiosos, pescadores artesanales, agricultores, carpinteros y amas de casa. Además de estas fuentes orales se hizo una revisión de documentos relacionado con la historia de Corn Island.

\section{RESULTADOS Y ANÁLISIS Primeros habitantes de Corn island}

Según Edward Conzemius, etnólogo francés de Luxemburgo, en 1929 afirma en su artículo Les Iles Corn Du Nicaragua" (Las Islas Corn de Nicaragua), que los primeros habitantes de Corn Island fueron los indigenas kukras, quienes fueron exterminados por los indígenas miskitos en alianza con los bucaneros ingleses. Estos utilizaban la Isla para reabastecerse de leña, agua, reparación de sus buques y como soporte para sus actividades de pesca.

Los colonos puritanos de origen británico llegaron a la isla a mediados del siglo XVIII, trayendo consigo sus esclavos de descendencia africana. Se sabe que vinieron desde Jamaica, estableciéndose en Honduras Británica (Belice) hasta llegar al litoral atlántico de Nicaragua.

Los primeros habitantes colonos habitaban preferentemente la parte Sureste de la isla. Esto tenía sus razones: La altura de Quinn Hill ofrecía una vista panorámica hacia el mar, desde donde podían visualizar la llegada de barcos piratas al acercarse a "Insurance Harbor", un puerto estratégico en la Isla, que brindaba facilidades para anclar y descargar las embarcaciones. Este puerto "Insurance Harbor" tiene, además, una importancia histórica ya que en él desembarcó el Coronel Alexander McDonald, superintendente de Honduras Británica (Belice), un 27 de agosto de 1841. Este superintendente fue quien mandó llamar a los esclavos, en el punto llamado South-West Bay, para declararlos libres de la esclavitud, en nombre de la Reina Victoria de Gran Bretaña y el Rey Robert Charles Frederick de la Mosquitia.

Después del establecimiento de los colonos ingleses en las islas de Corn Island se da inicio a un proceso de transculturación. La mayoría de los colonos llegaron a estas islas como aventureros y exploradores en busca de fortunas y riquezas. Se establecieron permanentemente interrelacionándose con los esclavos. Producto de esta relación surge la descendencia creole de Corn Island. En el transcurso del tiempo, esta descendencia fue asimilando y adaptando paulatinamente las costumbres y tradiciones de la cultura Inglesa. Es así como surge el perfil antrogenético de la etnia creole de Corn Island.

A partir de la emancipación de los esclavos el 27 de agosto de 1841, la mayoría de los colonos comienzan a abandonar Corn Island. Algunos regresaron a Europa y otros emigraron a las islas caribeñas: Jamaica, Bahamas, 


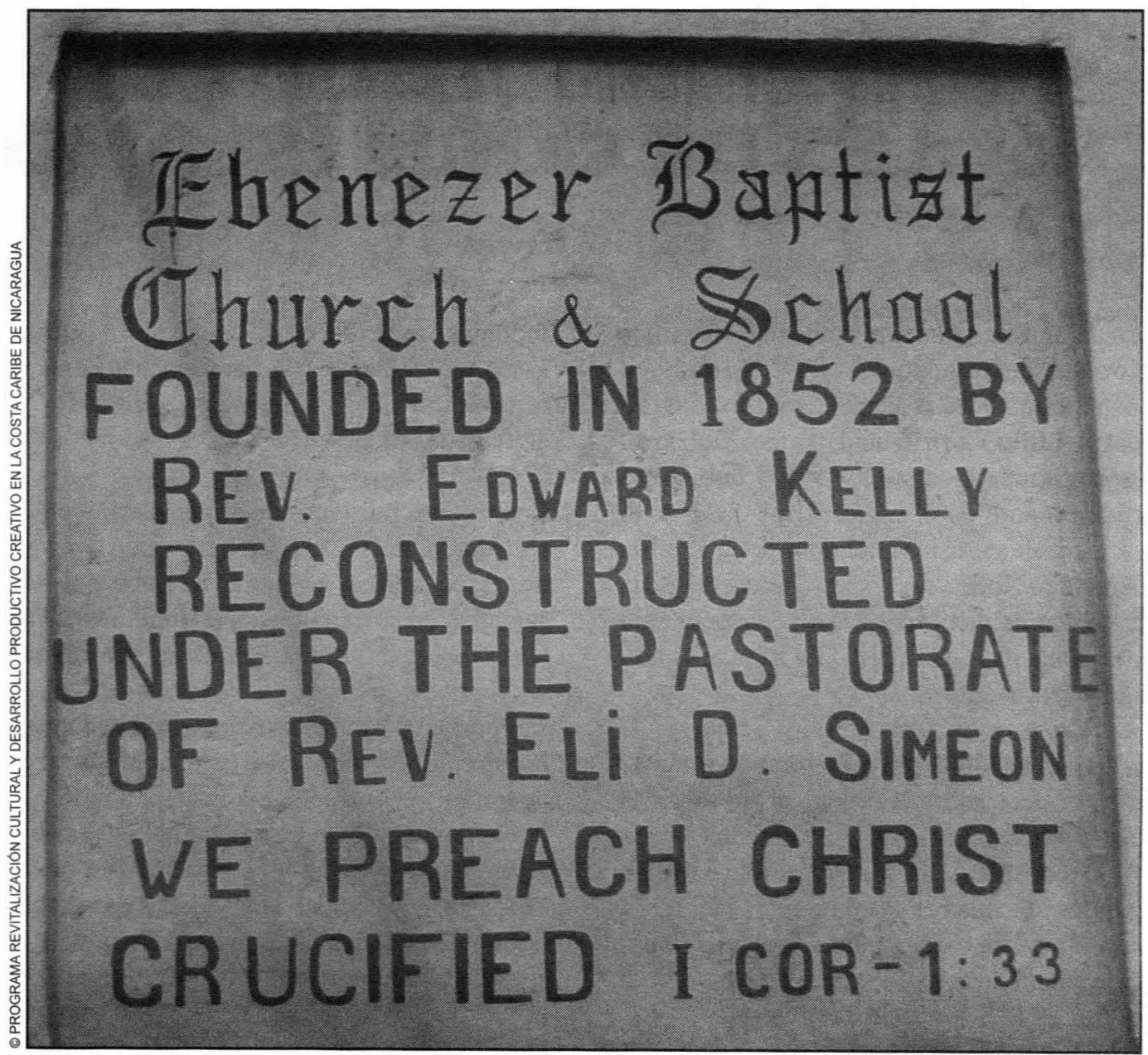

Iglesia Bautista Ebenezer. Corn Island 2011.

San Andrés, Providencia y Gran Cayman. Después de esta emancipación, la población libre comienza a cultivar la tierra para su propio beneficio y las familias del pueblo ancestral creole comienzan a consolidarse.

Con la emancipación en 1841, no termina la influencia inglesa en la Isla, ya que Corn Island, como el resto de la Mosquitia, sigue bajo la protección de Gran Bretaña a pesar de ser territorio nicaragüense. Esta condición permitió establecer una abierta y estrecha relación comercial entre Corn Island y las Islas Caribeñas, particularmente con San Andrés, Providencia, Jamaica y Gran Cayman.

Los apellidos de los creoles de Corn Island son de origen europeo, en su mayoría ingleses. Las primeras familias renombradas en la isla fueron los Quinn, Downs, Bowden, Forbes, Frances, Cottrell, Hunter, Hodgson, Handsanks, Brown, Hooker, Culver y Campbell. En la actualidad, los apellidos predominantes son Downs, Quinn y Campbell.
En un inicio, los barrios con mayor concentración poblacional eran North End y South End, posteriormente, a inicios de los años 60 , con la aparición de las fabricas procesadoras de langosta y otros mariscos en el barrio Brig Bay, este barrio comenzó a poblarse masivamente de migrantes y comerciantes, hasta convertirse en el día de hoy en el barrio de mayor concentración poblacional y territorial en el Municipio.

\section{Inicio de la Educación en Corn Island}

Según los portadores culturales de esta investigación, once años después de la emancipación de los esclavos arribó a la Isla el reverendo Edward Kelly, un maestro titulado y graduado de la Escuela Queen Street Baptist Day School en Belice. Este reverendo se encontró con una población creole que, en su mayoría, no sabía leer ni escribir. El hecho que el idioma predominante de la Isla fuera el inglés creole facilitó, al reverendo Kelly, poder iniciar su 
labor educativa, la cual serviría para el enriquecimiento y ampliación de los conocimientos de la población y para facilitar al Reverendo las enseñanzas del evangelio. Con el transcurso del tiempo, la influencia del reverendo Kelly fue creciendo de tal manera que pudo lograr el establecimiento de la primera escuela formal de Corn Island, en los predios de la misión Bautista del barrio de North End.

Los ancianos entrevistados narran que producto de esta labor de enseñanza fue que el reverendo Kelly pudo establecer la primera institución religiosa en Corn Island: la iglesia bautista Ebenezer la cual comenzó a funcionar con el reverendo Kelly a la cabeza y sus destacados alumnos. Estos comenzaron a asumir cargos dentro de la estructura de la incipiente Iglesia. En estas labores se destacaron los alumnos Isaiah H. Lampson, Michael Quinn, Simeon Brooks y Amon Hodgson, entre otros.

Durante el periodo 1853-1880, el reverendo Kelly desarrolló una ardua labor de enseñanza en conjunto con sus primeros estudiantes quienes sirvieron como promotores de educación durante estas tres décadas. Los resultados fueron extraordinarios, ya que durante el periodo de 1880 la mayoría de los adultos en la Isla ya sabían leer y escribir. A partir de 1880, el Reverendo comenzó a dedicar mayores esfuerzos al desarrollo y consolidación de la iglesia bautista Ebenezer, sin descuidar la escuela ya establecida dentro de los predios de la Iglesia.

Según nuestros entrevistados, de igual manera se logra establecer, en el barrio South End, una escuela privada dirigida por el Sr. William B. Morgan quien se había destacado en la labor de enseñanza en Kingston Jamaica. Según relata el reverendo Rodwell Morgan en su libro Meet Corn Island, esta escuelita privada funcionó por un periodo de tiempo. En 1895, por primera vez, en este municipio se recibe apoyo educativo por parte del gobierno de Nicaragua. Esta ayuda consistió en el pago de diez pesos mensuales por el alquiler del centro dominical de la iglesia Bautista de South End, para desarrollar las labores de enseñanzas del profesor William Morgan. Esta escuelita funcionó por algunos años en este local, posteriormente fue trasladada a la escuela bautista Ebenezer, de North End, junto con los alumnos.

La educación continuó desarrollándose paulatinamente y cada año se iba anexando un nivel más de enseñanza conforme el avance de los alumnos y el crecimiento de la demanda de la población estudiantil. Toda la enseñanza impartida a los alumnos, desde 1852 a
1945, fue en lengua materna (inglés). No fue sino hasta 1946 que la educación formal comenzó a impartirse en idioma español, con el apoyo del Gobierno a través del Ministerio de Educación.

La Iglesia Episcopal desempeñó su papel en el desarrollo de la educación. Establecieron y fundaron en el barrio de South End la escuela San Santiago bajo la dirección del reverendo Edward Hawkins en el año 1939.

La Escuela Superior Mixta de Corn Island fue fundada en 1950 por la profesora Essie Nixon. Dicha escuela fue reconocida por el Ministerio de Educación convirtiéndose en la primera escuela pública de Corn Island.

La escuela General Rigoberto Cabezas se empezó a construir en 1969, en el mismo lugar donde estaba ubicada la Escuela Superior Mixta en el barrio North End. El 27 de agosto de 1970, en horas de la mañana de un día lluvioso se inaugura la Escuela Rigoberto Cabezas bajo la dirección de la profesora Rowena Luzey Ow.

El Instituto Nacional Alva Hooker Downs, el primer instituto de secundaria en Corn Island, fue fundado por la profesora Alva Hooker Downs, cuando la Escuela Bautista Ebenezer de secundaria tuvo que cerrar en 1969. Cuentan que La profesora Alva Hooker Downs, profesora nativa de Corn Island, al ver la caótica situación de la educación se moviliza y aprovechando sus influencias logra obtener el financiamiento del Gobierno para la construcción de un instituto. En 1975 comenzó a funcionar el Instituto Nacional de Corn Island (I.N.C.I.) que dignamente hasta esta fecha lleva el nombre de su fundadora: Instituto Nacional Alva Hooker Downs (INAHD).

Según el Sr. McNeill Bryan, la Iglesia Ebenezer reinicia sus enseñanzas de escuela secundaria, en 1994, con la apertura de una escuela cuya dirección estuvo a cargo de la profesora Marie Rigby Downs. Este colegio logra efectuar la primera graduación de bachilleres en Ciencias y Letras en 1999. Hay que reconocer los créditos de este logro a los esfuerzos y la ardua labor de la fundadora de esta escuela: profesora Marie Rigby Downs.

Otras escuelas de primaria también han contribuido a la enseñanza. Según nos comentó el Sr. Vance Quinn, uno de nuestros portadores culturales, a partir de los años cincuenta hasta la fecha tenemos la escuela Católica, la escuela Bautista Trinity, la escuela Essie Nixon, la escuela Olive Brown, la escuela Hedly Wilson, la escuela Fay Angels la escuela la islita y la escuela Nubia Rigby. 


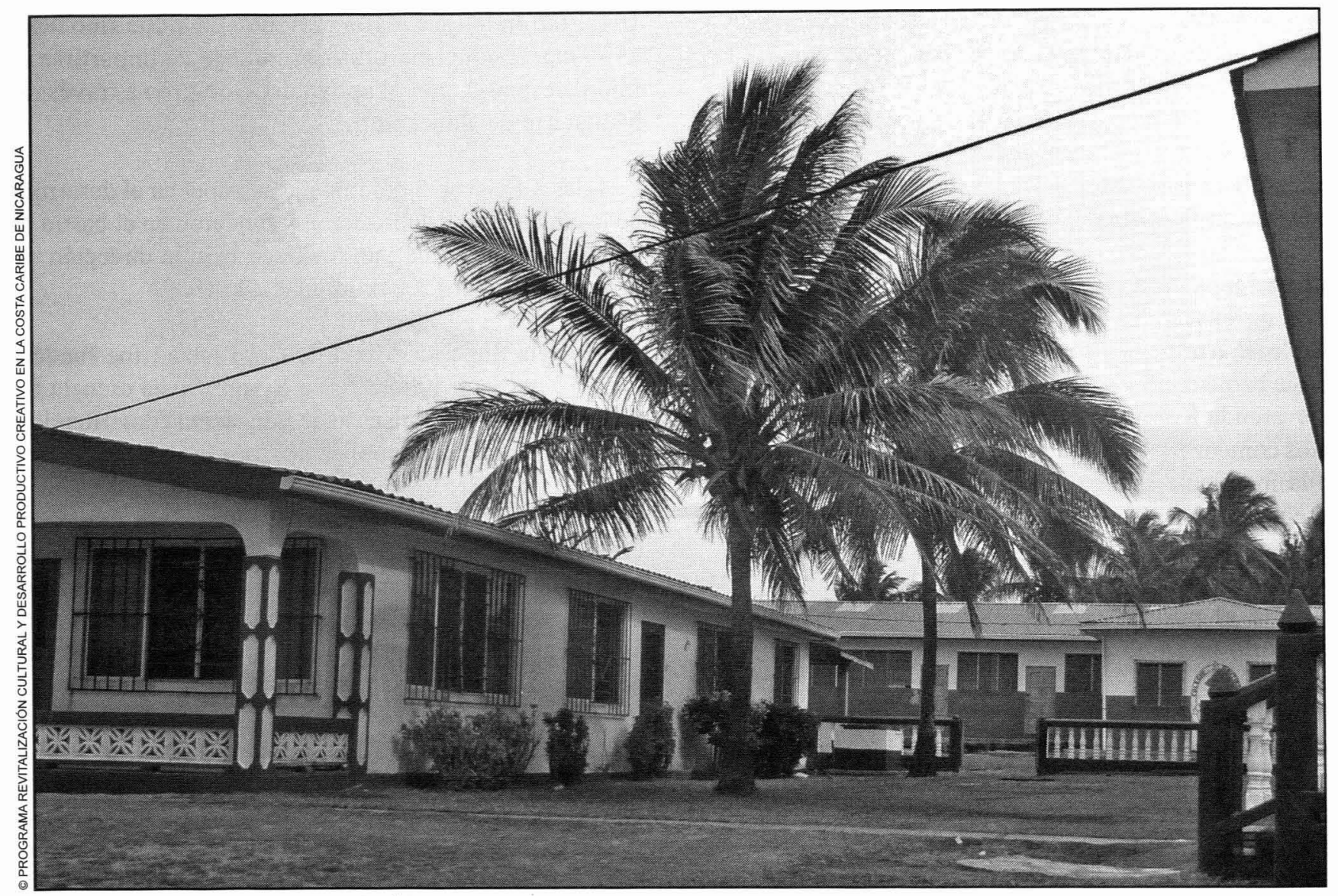

Escuela-Iglesia Morava. Corn Island 2011.

En el año 2000, la Bluefields Indian and Caribean University (BICU) inaugura un modulo en Corn Island, bajo la dirección de la Lic. Gay Downs Halford. Luego, en el año 2005, bajo la dirección de la Lic. Delia Downs White se gradúan los primeros 14 alumnos en la Licenciatura de Psicopedagogía. Después, esta universidad ofrece las carreras de Administración de Empresa con énfasis en Hotelería y Turismo, bajo la Dirección de la Lic. Arlene Hodgson Downs. El 25 de noviembre del año 2010 se realizó la segunda graduación con 15 nuevos licenciados en Administración de Empresa con énfasis en Hotelería y Turismo.

\section{La Religión}

Según Canzoneri (1977), el primer misionero que vino a esta comunidad fue el reverendo Edward Kelly. En el año 1852 estableció la Iglesia Bautista Ebenezer en el barrio de North End. El misionero utilizó este establecimiento inicialmente para enseñar a leer y escribir a los destacados líderes naturales de la comunidad. Estos sirvieron como multiplicadores del aprendizaje, tanto para la lectura como para la escritura y también para predicar la palabra de Dios. El éxito de estas enseñanzas fue tan marcado que, una década después, el reverendo Kelly pudo conformar la primera congregación de la Iglesia Bautista Ebenezer, destacándose entre ellos sus primeros alumnos cuyos nombres fueron: Harold Isaiah Lampson, Michael Quinn, Simeon Brooks, Frank Campbell, Amon Hodgson, Peter Lampson, Auntie Cinda, entre otros. La iglesia Bautista fue fortaleciéndose paulatinamente, convirtiéndose en la más importante de todas en la comunidad creole de Corn Island.

Los misioneros moravos hicieron su primera visita a esta comunidad en 1858 , pero obtuvieron poca aceptación de la población. No obstante continuaron con sus esfuerzos de establecerse. En 1875 realizaron los últimos intentos, sin embargo, cinco años después, cuentan los portadores culturales entrevistados, abandonaron la isla por un largo periodo.

Debido al auge económico que produjo el inicio de la pesca, explotación y comercialización de langosta, que generó una gran demanda de mano de obra, muchos miskitos migraron 
masivamente hacia Corn Island, en busca de trabajo, y se establecieron en el barrio Brig bay. Estos migrantes venían de la zona norte de la Costa Atlántica, donde la religión morava era predominante.

En 1965, por petición de la población miskita establecida en el barrio Brig Bay, la Misión morava reinicia su labor. Esta vez se establecieron permanentemente en Corn Island. Las ceremonias y misas dominicales se celebraban en lengua miskita, ya que los miembros y la congregación eran netamente de esta etnia. Con el transcurrir de los años, la población creole que se identificaba con la iglesia morava asistía a la misa dominical. Esta población fue creciendo, y a mediados de los años noventa e inicio del dos mil, la población creole logra conformar su propia congregación que hoy en día funciona en su propia iglesia en el barrio de Brig Bay.

Según el Rev. Allan Taylor, a finales del siglo XIX, los misioneros anglicanos comenzaron a visitar la isla, con la finalidad de establecerse en esta comunidad. El día 1 de mayo de 1901 se asienta la misión anglicana en el barrio South End. La congregación de la Iglesia estaba conformada por los habitantes creoles del barrio de South End. Esta misión religiosa fue la fundadora de la escuela San Santiago, en el año de 1939, dirigida por el padre Edward Stanley Hawkins.

\section{Actividades festivas de la emancipación}

Según relatan nuestros portadores culturales entrevistados, las actividades festivas en conmemoración de la emancipación de los esclavos fueron una iniciativa de Edward Kelly, pocos años después de haber fundado y establecido la Iglesia Bautista Ebenezer. Probablemente en el año 1859.

Estas primeras celebraciones se dieron en el patio de la señora Teresa Lampson, bajo un imponente y frondoso árbol de higuera durante todo el día del 27 de agosto. La celebración consistía primeramente en una misa de acción de gracias; posteriormente se realizaban juegos de kitty ally, cricket, chibolas, carreras de caballos, maratones y competencias de carrera, entre otras. El almuerzo era preparado por las mujeres miembros de la Iglesia. Había sopa de cangrejo, carne de cerdo y de cabra, acompañado de bastimentos y queque de yuca, plátano, banano y patata. Los niños recitaban versos de la Biblia mientras los adultos cantaban himnos. Así y de esta manera continúa celebrándose este acontecimiento.

Al fallecer la señora Lampson, la celebración de esta festividad fue trasladada a diferentes patios o predios de miembros de la congregación bautista, y posteriormente en el patio de la iglesia. Para entonces había más personas involucradas. Todos los isleños participaban, y de esta manera se lograba mayor acercamiento entre los habitantes de todos los barrios y la conformación de una comitiva para la celebración de esta festividad. Luego, cada familia buscaba la manera de aportar algo, ya sea un cerdo, una gallina, una vaca, bastimento, refresco naturales, lo que estaba a su alcance, para poder ofrecer o llevar a la iglesia para ese gran día 27 de agosto.

Las mujeres estaban encargadas de preparar el alimento, de servirlo y de cuidar a los niños. Los hombres de recoger las comidas en las casas donde eran preparadas y trasladarlas en un bote de remo al patio de la iglesia. En la hora del almuerzo se servía primero a los más viejos, luego a los niños y después a los demás. La comida se daba en hojas de uvas marinas y la sopa de cangrejo en una calabash (guacal). Cuando todos estaban servidos, el pastor o un líder de la iglesia bendecía la comida y seguidamente todos podían comer y disfrutar del encuentro. Después del almuerzo había soda cake y ginger beer y otros tipos de queques hechos todos en cacerolas. Esta era la oportunidad que tenían los niños de correr al mar y darse un chapuzón.

Al finalizar esta celebración en la tarde, todos conjuntamente limpiaban la iglesia y el patio dejando todo en su lugar. Luego se cantaba un himno de despedida y la oración al final. Por muchos años, los predios de la iglesia bautista Ebenezer fue el escenario de estas festividades. A través de los años estas actividades festivas se continuaron realizando pero con mayor creatividad, incorporando a los eventos la carrera de cintas a caballo, para escoger y seleccionar a la reina del evento del 27 de agosto. A partir de la década de los ochenta, las festividades conmemorativa de la emancipación de los esclavos se trasladó al barrio de South End y las autoridades municipales comienzan a involucrase directamente en la conducción de esta festividad que poco a poco se ha ido comercializando y perdiendo su autenticidad, el contenido de sus principios, valores morales y éticos, que reinaban en estas festividades celebradas por nuestros ancestros.

\section{Aspectos políticos}

Durante el periodo de gobierno de La Mosquitia, Corn Island pasó a ser parte de la jurisdicción de Bluefields, y el jefe de La Mosquitia tenía la facultad de nombrar las autoridades de Corn Island. En un inicio, el gobierno de la Mosquitia asignó a un funcionario de Bluefieds para ejercer el cargo de juez en la isla. La población 
estaba inconforme con el funcionamiento del gobierno de La Mosquitia y tomaron el nombramiento del Juez de Bluefields como una imposición, protestaron este hecho ante las autoridades nicaragüenses y no fue hasta en el año 1890 cuando el Gobierno nombra directamente para Corn Island al señor Michael Quinn como juez, en respuesta a las protestas que por años venían haciendo los isleños. El señor Michael Quinn, según los relatos, era uno de los líderes más destacado en la comunidad. Era quien hacia las labores de médico, dentista, carpintero, miembro activo de la iglesia bautista, entre otras cosas.

Según el reverendo Rodwell Morgan (1996), después de asumir el general José Santos Zelaya la presidencia de la república en 1894, el general José Santos Zelaya, el primer gobernador nombrado en Corn Island fue el señor Jacob Chapman quien era residente en esta isla. A partir de este periodo, los gobernadores hacían la función de alcalde, jefe de policía y encargado del registro público. El segundo gobernador fue el señor Adalphus Downs, originario de Corn Island, quien estuvo al frente del gobierno municipal por un largo tiempo. Durante este periodo se organizaron mejor las estructuras del gobierno municipal. Se nombró delegados representantes del gobernador en cada uno de los barrios. Estos delegados tenían bajo su responsabilidad el velar por la buena conducta y el orden en su barrio. Los problemas menores eran resueltos a nivel del barrio y la palabra y decisión del delegado era ley. Eran conocidos popularmente como "Cantan Judge".

Dentro de esta estructura de gobierno municipal, también había un secretario nombrado por el gobernador que tenía bajo su responsabilidad el registro civil de la personas. Durante esta época, los delegados del gobernador en los barrios de mayor renombre (Cantan Judge) fueron los señores Charles Downs, Benjamín Winrights (barrio de South End), Lucius Bent, Daniel Brooks (barrio de North End), Cantan Judges, del barrio de South End, Rodley Hunter, Cayetano Hunter, Thomas White y Freddy White.

En 1936 asume el poder el gobierno liberal encabezado por el Gral. Anastasio Somoza García quien militarizó todo el territorio nacional nombrando a jefes políticos en los distintos departamentos del país, sustituyendo a los gobiernos civiles en los departamentos. En el caso de Corn Island enviaron a un teniente de la guardia nacional quien hacia las labores como un agente policial, sustituyendo el cargo del gobernador en muchas circunstancias. Corn Island en 1940 fue declarado oficialmente un nuevo municipio dentro del departamento de Zelaya. Producto de estos nuevos cambios en la estructuras de la política gubernamental es nombrado, el nueve de febrero de 1940 , el Sr. Isaiah Harold Lampson Jr, como el primer alcalde del municipio de Corn Island. (alcaldía de Corn Island, y portadores culturales)

Según información brindada por la alcaldía de Corn Island, los alcaldes asignados al cargo posteriormente fueron:

$\begin{array}{ll}\text { Sr. Obidaiah White } & 1945-1947 \\ \text { Sr. Harry Quinn } & 1947-1948 \\ \text { Sr. Ferdinand Nicholson } & 1948-1961 \\ \text { Sr. Marvin Wrights } & 1962-1963 \\ \text { Sr. David Richards } & 1963-1966 \\ \text { Sr. Norman Downs } & 1967-1969 \\ \text { Sr. Isaiah H Lampson } & 1969-1972 \\ \text { Sr. Cordell Nicholson } & 1979-\text { julio-1979 } \\ \text { Sr. Orvell Morgan } & \text { julio-1979-nov-1979 } \\ \text { Sr. Cleaveland Webster } & 1979-1980 \\ \text { Sr. Hurley Morgan } & 1980-1988 \\ \text { Sr. Ena Moses Hart } & 1989-1996\end{array}$

Los alcaldes elegidos por el pueblo a través del voto en conformidad con la Constitución de la República:

Sr. George Howard

$1997-2000$

Sr. Roberto Ow

2001-2004

Sr. Alex Dixon

2005-2008

Sr. Cleaveland Webster

2009-2012

A partir de 1940 , según los portadores culturales entrevistados, jueces de esta localidad eran nombrados directamente por el gobierno central. Durante esta etapa, entre 1940 y 1979, los jueces que asumieron esta responsabilidad eran originarios de Corn Island, entre ellos se destacan los señores Carl Jackson, Angus Quinn, Felix Britton, Dacosta Bodden y Limecio Ow, La Sra. Lorraine Bodden, Lic. Perla Coleman, Lic. Julissa Downs, la Lic. Silfrides, Lic. Teresa Peña y la Lic. Carmen Merlo.

Desde 1979 hasta la fecha, la estructura municipal de gobierno ha ido cambiando y adaptándose a las estructura de las otras municipalidades dentro del territorio. El Ejército, con su propia estructura; de igual manera, la Policía Nacional; la Alcaldía Municipal funcionado dentro de un edificio, con las distintas áreas como el resto de las 


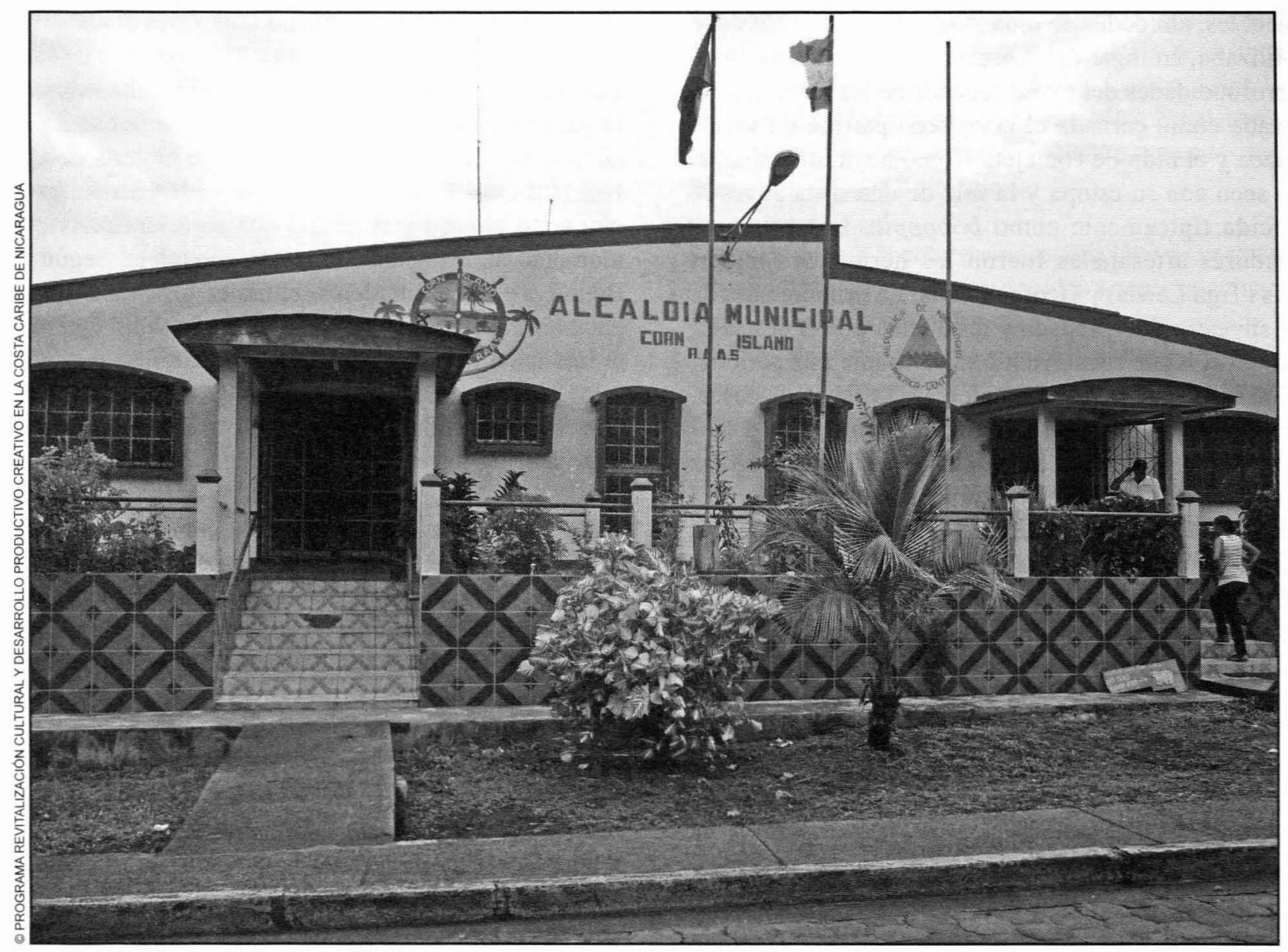

Alcaldia Municipal. Corn Island 2011.

municipalidades; un juzgado con sus propias instalaciones, la delegación del Ministerio de Educación con su propio edificio y personal docente, un centro de salud con sus propias instalaciones y la presencia permanente de médicos y enfermeras para atender ambas islas.

\section{Aspectos Económicos}

Después de la emancipación de los esclavos, según los relatos de los portadores culturales, la población creole se dedicaban a la agricultura, crianza de animales domésticos y la pesca para el auto consumo. La agricultura, debido a la fertilidad de la tierra por su origen volcánico, al poco tiempo se convirtió en la fuente de ingreso más importante de la comunidad. Esto les permitió establecer relaciones comerciales de intercambio de productos entre San Andrés, Providencia, Gran Cayman y Jamaica. Los productos agrícolas de mayor importancia eran el coco, plátanos, cítricos, tubérculos y raíces. La crianza de animales domésticos se utilizaba principalmente para el auto consumo.
La pesca: en un inicio, la pesca de escama, de langosta y tortuga era exclusivamente para el auto consumo, pero en la medida en que se fue fortaleciendo la actividad comercial en el Caribe y el resto del litoral atlántico, esta actividad fue convirtiéndose poco a poco en una de las actividades económicas de mayor importancia dentro del Municipio. Los señores Vance Quinn y Abraham Forbes nos relatan que a mediados de los años cincuenta e inicio del sesenta, los hermanos Bowers, de nacionalidad norteamericana, dieron apertura a la compra y procesamiento de langosta en Corn Island. Esta actividad comercial la iniciaron a bordo de la embarcación "J.B." Comenzaron pagando un costo de 50 centavos córdobas por cada langosta, al poco tiempo después incrementaron el precio a un córdoba por langosta entera.

Los incipientes pescadores artesanales inician sus actividades pesqueras con la construcción de nasas fabricadas de caña silvestre (wild cane) que para en ese entonces abundaba en los suampos, pantanos y 
humedales, alrededor de toda la isla. El bejuco silvestre se utilizaba, en lugar del mecate, para jalar las nasas de las profundidades del mar alrededor de los arrecifes. Se utilizaba como carnada el coco seco partido en varios pedazos y el nido de comején. Como boya utilizaban el coco seco con su estopa y la raíz de una fruta silvestre conocida típicamente como bobapple. Los primeros pescadores artesanales fueron los hermanos Shubert Lewis (Tiga Lewis) y George Lewis. La primera nasa de caña silvestre fue diseñada y producida por el pescador artesanal el señor Yucal Archibold. Durante este periodo, cada pescador utilizaba, para efectuar su faena de pesca, entre tres a nueve nasas. Realizaban entre dos a tres faenas cotidianamente. La producción de langosta estaba en su apogeo y el costo de producción era mínimo. El pescador artesanal que tenía mayor cantidad de nasas a su disposición en los años sesenta era el señor Shedrack Gordon quien operaba con treinta y seis nasas.

A inicio del siglo veinte, el coco significó ser el producto más importante de la economía y sobrevivencia de los habitantes de la Isla. Cada familia tenía sus propias tierras y trabajaban individualmente en la siembra y recolecta de su cosecha. La islita (Little Corn Island) era usada por los agricultores principalmente para la siembra de coco, plátanos, raíces y la crianza de ganado y cerdos. El medio de transporte que se utilizaba entre las dos islas eran barquitos de vela; y a lo interno de las islas, como medios de transporte se utilizaban mulas y caballos.

En 1960, la cosecha de coco comenzó a convertirse en un producto de menor importancia económica, siendo sustituida gradualmente por la pesca de langosta ya que la misma generaba mayor ingreso económico a las familias. El Municipio se desarrolló, desde el punto de vista social y económico, con las instalaciones de las empresas procesadoras de mariscos. A mediados de los años sesenta generaron mayores demandas de servicios de infraestructura, mano de obra, y el servicio de técnicos y profesionales. La migración de nuevos habitantes, en su mayoría miskitos, permitió resolver el problema de la mano de obra para las embarcaciones de la empresa y el procesamiento de langosta. Además, para los agricultores, los miskitos migrantes fueron una respuesta para el problema de limpieza y recolecta de la cosecha, ya que, durante este periodo, la gran mayoría de la población se dedicaba exclusivamente a la pesca, por su rentabilidad. A partir de 1975, la actividad pesquera se convirtió en la más importante del Municipio, desde el punto de vista económico, hasta la fecha de hoy.

\section{Salud}

Cuenta Miss Bonnilyn Tucker Quinn, con una sonrisa que la hace aparentar mucho menos edad que sus actuales 86 años, que ella es descendiente de los Quinns de Quinn Hill (La Loma). Al referirse a sus antepasados, lo hace con tanto orgullo y nostalgia que pareciera revivir cada momento de los acontecimientos que relata. Según Miss Bonnilyn y otros portadores culturales, los Quinns nacieron para ser trabajadores de la Salud. El Sr. John Quinn, con la intención de ayudar a su comunidad optó por atender a los enfermos consultando libros y textos de medicina que fueron proporcionados por un médico del buque de guerra británico que, en ocasiones, pasaba por la Isla dando servicio a pacientes enfermos de la comunidad. El señor John Quinn, debido a su pasión por la medicina y a la asimilación de lo que había aprendido de los libros se convirtió en el primer médico autóctono del pueblo creole de Corn Island. Durante este periodo utilizaba plantas medicinales y remedios caseros obtenidos de nuestra vegetación.

El señor Quinn heredó sus conocimientos y libros de medicina a su hijo Michael Quinn y a sus seis hijas, Susan, Margaret, Martha, Catherine, Elizabeth y Mary. El Sr. Michael Quinn, conocido cariñosamente por los pobladores de la isla como "Uncle Mike", desempeñaba las funciones del nuevo médico de la comunidad. El hacía sus visitas rutinarias a caballo, atendiendo enfermos y funcionando como dentista cuando era necesario.

Cuentan que este señor extraía muelas sin anestesia, lo único que estaba disponible para amortiguar un poco el dolor eran las bebidas alcohólicas caseras. Con todas estas limitaciones, este noble servidor siguió ejerciendo su vocación de salvar vidas. Relata el Rev. Allan Taylor que -dicen- cuando "Uncle Mike" atendía a un paciente y las medicinas y remedios caseros no daban resultado ya que el paciente continuaba gravemente enfermo, entonces utilizaba su último recurso: una sola pastilla que era conocida con el nombre de "One and Only" (una solamente). El paciente se recuperaba o moría después de esta dosis.

El señor Michael Quinn instruyó al Sr. Clinton Quinn en la extracción de muelas, quien lo hacía muy eficientemente después de la muerte del Sr. Quinn en el año 1938. Las seis hermanas Quinn eran parteras, pero además tenían la habilidad y el don de sobar y corregir cualquier tipo de fracturas o problemas de los huesos y tendones. Durante muchos años, esta familia trabajó y luchó en beneficio de la salud del pueblo creole de Corn Island. 
Continuando Miss Bonnilyn su narración afirma que también hubo otras mujeres que se destacaron como enfermeras, especialmente en el área de labor y parto, entre ellas, Miss Sylvia Welcome, nativa de Corn Island. Con la vocación de servir a su comunidad y con la ayuda y apoyo de sus padres decide ir a Jamaica para estudiar Enfermería. $\mathrm{Al}$ regresar trabaja como enfermera.

Después de la muerte del Sr. Maichael Quinn, quien por muchos años fue el único doctor de la Isla, a pesar de no tener título de doctor, pero con vocación, dedicación, perseverancia y amor hacia sus semejantes. Siguieron los pasos de Uncle Mike los señores Obidiah White y Carl Jackson, aunque no tan eficientes como uncle Mike, pero sí aportaron y ayudaron a muchos enfermos, inclusive, en algunas ocasiones salvaron vidas. Cuentan los portadores que al Sr. Jackson le decían el "Doctor Penicilina", porque para todo recetaba inyección de penicilina. No importaba si el enfermo fuese niño $o$ adulto. El Sr. Obidiah White era conocido como The Island Doctor. También fue líder en la Iglesia Bautista, y en ocasiones conducía la misa dominical.

Cuenta la Sra. Lorna Hodgson que durante esta época se destaco la Sra. Martha Quinn Downs, conocida popularmente como "Big Mama". Ella vivía en el barrio de North End, atendiendo a cualquier persona que tenia fractura y dislocación de huesos. De igual manera había otras mujeres que se dedicaban a servir a la comunidad en las diversas labores de salud, entre ellas se destacaban la Sra. Hortense Ebanks, la Sra. Letisha Downs, la Sra. Martha Downs y la Sra. Ellen White. Y como parteras, las Sras: Elsie Rodríguez, Rizona Windrights, Elora Campbell, Merlin Willson, Imogene White, entre otras.

Otra enfermera nativa de Corn Island fue Mrs. Casilda Morgan Downs. "Miss Cassie", como era llamada cariñosamente por los isleños. Trabajó más de veinte años sirviendo a la comunidad creole. Su casa de habitación se convirtió temporalmente en una clínica, pero también atendía a pacientes, aun en altas horas de la noche, en sus casas sin importarle en qué parte de la isla vivían. Después de muchos años de servicio en su pueblo natal de Corn Island, Miss Cassie se traslada con su familia a la ciudad de Bluefields, dejando en su lugar a Miss Ethel Kandler a quien había instruido en labor y parto. Miss Ethel kandler resulto ser una excelente partera, atendiendo a centenares de embarazadas y partos. Aun cuando había una clínica con un médico y una enfermera, las mujeres preferían ser atendidas por Miss Ethel en su casa. Cabe mencionar que esta noble mujer cobraba simbólicamente por sus servicios, algunas simplemente le daban las gracias. Esta fiel servidora siguió ejerciendo con devoción su labor. Miss Ethel fue un fiel miembro de la Iglesia Bautista Ebenezer y miembro del coro de la Iglesia. Cuenta Miss Lorna Hodgson, portadora cultural, que Miss Ethel tenía una melodiosa voz y que actuaban juntas en dramas y recitales en las actividades de la Iglesia.

Después de que la enfermera Casilda Downs se traslada a Bluefields hubo ciertas inquietudes y preocupaciones por parte de la población, ya que la atención médica había disminuido por la falta de personal. Estas preocupaciones fueron retomadas por el Padre Davis de la Iglesia Episcopal, quien aprovechándose de sus influencias logra obtener el financiamiento para la instalación de la primera clínica de Corn Island la cual tenía el nombre de Saint James (San Santiago) y estaba ubicada en el barrio de South End en el año 1962.

Esta Clínica fue supervisada desde Puerto Cabezas por el Dr. Ned Wallace, ya que en ese entonces no tenían médico permanente. Según el Sr. Rincard Hodgson, en aquel momento, la Enfermera Doris Timmins, junto con la enfermera auxiliar Mescal Campbell, estaban al cuidado de la clínica y de los pacientes. Luego, una enfermera titulada de Bluefields, de nombre Yolanda Campbell, se incorporó a esta noble causa y juntas trabajaron apoyando y velando por la salud de la comunidad.

Pocos años después vino de Bluefields el Dr. Thomas Hodgson como médico oficial de la Clínica Saint James. Este médico era de origen creole. Tuvo mucha aceptación y fue muy querido por la población isleña. Trabajó varios años en la comunidad junto al enfermero Rincord Hodgson, el Sr. Allan Taylor, actual Padre de la Iglesia Episcopal de Corn Island, y la enfermera auxiliar Rose Downs, todos nativos del Municipio. Cuando el Dr. Hodgson se traslada a Bluefields a continuar con sus labores medicas, el enfermero Sr. Allan Taylor tuvo que desempeñar la función de médico bajo la supervisión del Dr. Ned Wallace. El enfermero Taylor desempeñó eficientemente su papel como médico, salvando vidas y curando a muchos enfermos con diferentes síntomas y enfermedades. Su fiel servidor, el enfermero Taylor siguió atendiendo a pacientes en su casa de habitación al igual que lo hacía con las visitas domiciliares.

Cuentan los portadores culturales que el Dr. Hodgson, aprovechando sus influencias y la amistad que tenia con el Dr. Cantón comenzó a gestionar, a través del Gobierno, la construcción de un hospital para Corn Island, pero no obtuvieron la aprobación ya que argumentaron que la 


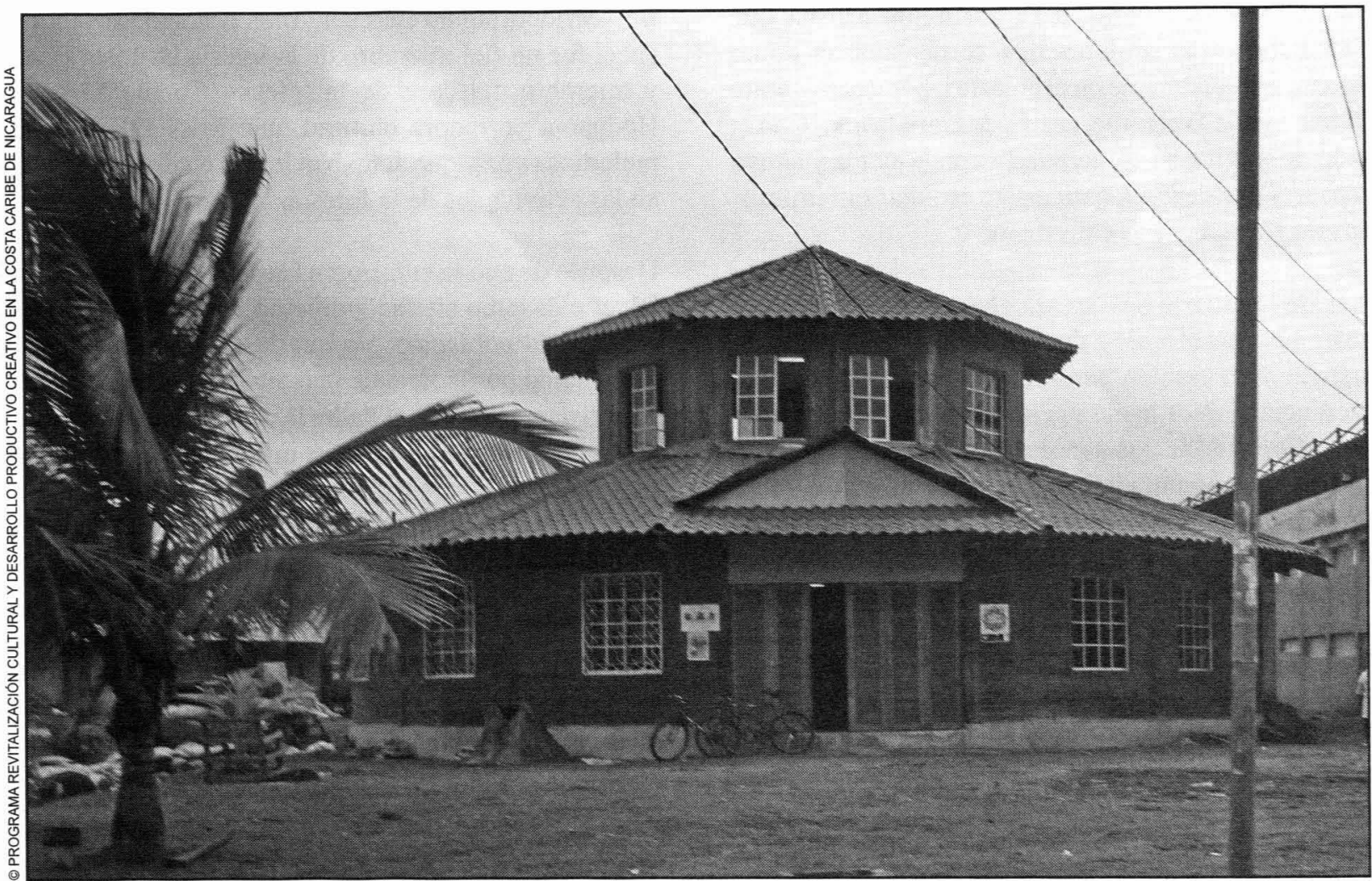

Casa de Cultura . Corn Island 2011.

población era demasiado pequeña para una inversión de esa magnitud. Sin embargo lograron obtener el financiamiento y la construcción de un centro de salud en el barrio de North End, en el año 1969, el cual fue inaugurado el 27 de agosto de 1970.

Nuevamente regresa a Corn Island el Dr. Thomas Hodgson, con la finalidad de trabajar y estar a la cabeza del funcionamiento del nuevo centro de salud. El personal creole nativos que trabajó en este centro de salud por muchos años fueron las enfermeras auxiliares Julieta Downs, June Britton, Rincord Hodgson, Nubia Downs, Elormis Hoy, Dr. Ricardo Taylor entre otros. A pesar de que esta clínica comenzó a atender a la población, la gente continuaba buscando al enfermero Allan Taylor para que los atendieran como médico a pesar de no tener un título como tal. Se había ganado el respeto y la confianza de la comunidad para resolver sus problemas de salud, independientemente de que ya la clínica como tal no estaba funcionando por problemas financieros, y por ser un establecimiento privado no contaba con la ayuda ni la solidaridad del Gobierno. Esto fue un duro golpe para los habitantes creoles, ya que esta clínica Saint James fue una bendición de Dios, que por muchos años representó el alivio y solución a un sin número de problemas de salud.
El centro de salud siguió funcionando en el barrio de North End hasta el año de 1988. Después del huracán Joan, que destruyó totalmente las instalaciones del centro de salud en North End, se tuvo que trasladar el centro de salud Ethel Kandler en el barrio de Brig Bay que aun no se había inaugurado ya que hacía falta completar y concluir con las instalaciones.

El Sr. Iriod Ruiz, portador cultural y presidente de la comisión de salud del Centro de Salud Ethel Kandler, nos cuenta que durante la administración de la alcaldesa Ena Moses Hart llegó a la isla una donación para la construcción de un hospital, por parte de los italianos. La alcaldesa hizo todo lo humanamente posible para lograr concretizar la ejecución y materialización de este proyecto. Logró finalmente comprar el terreno y posteriormente la construcción de este nuevo centro de salud con dos pabellones y amplios espacios para poder extender las construcciones en el futuro. Este Centro de Salud Ethel Kandler aumentó su personal y la nueva dirección estaba a cargo de la Dra. Lourdes Gutiérrez Nicholson, nativa, y después, del Dr. David Somarriba Watts también nativo de Corn Island. A través de los años, la isla ha producido sus propias enfermeras que han laborado en el centro de salud, entre ellas, Cándida Fuller, Wendy Downs, Ethel Williams, Lira Gómez y Margina Bent. 
El nombre de este centro de salud es en memoria de una partera que trabajó incondicionalmente con todas las mujeres que daban a luz en esta comunidad. Por varias décadas desempeñó de manera brillante y con mucha devoción la labor de parto en esta municipalidad. En la actualidad, este centro funciona bajo la dirección de la Dra. Fay Downs Halford, con seis médicos profesionales, diez enfermeras auxiliares, dos técnicas de laboratorio y otros trabajadores de la salud.

En 1981, con la iniciativa de la enfermera auxiliar Miss Nubia Downs Zamora se organizó como brigadistas de salud a un grupo de pobladores en los diferentes barrios de la localidad. Esta organización ha servido durante más de treinta años como el organismo básico y elemental en las exitosas jornadas de salud realizadas en diferentes momentos y circunstancias dentro del Municipio. En otras palabras, los brigadista de salud han sido la columna vertebral para el buen funcionamiento del centro de salud Ethel Kandler. Otra organización que ha servido al centro de salud es la Comisión de Salud. Esta comisión fue fundada en los años noventa, con el fin de apoyar y soportar el buen funcionamiento del

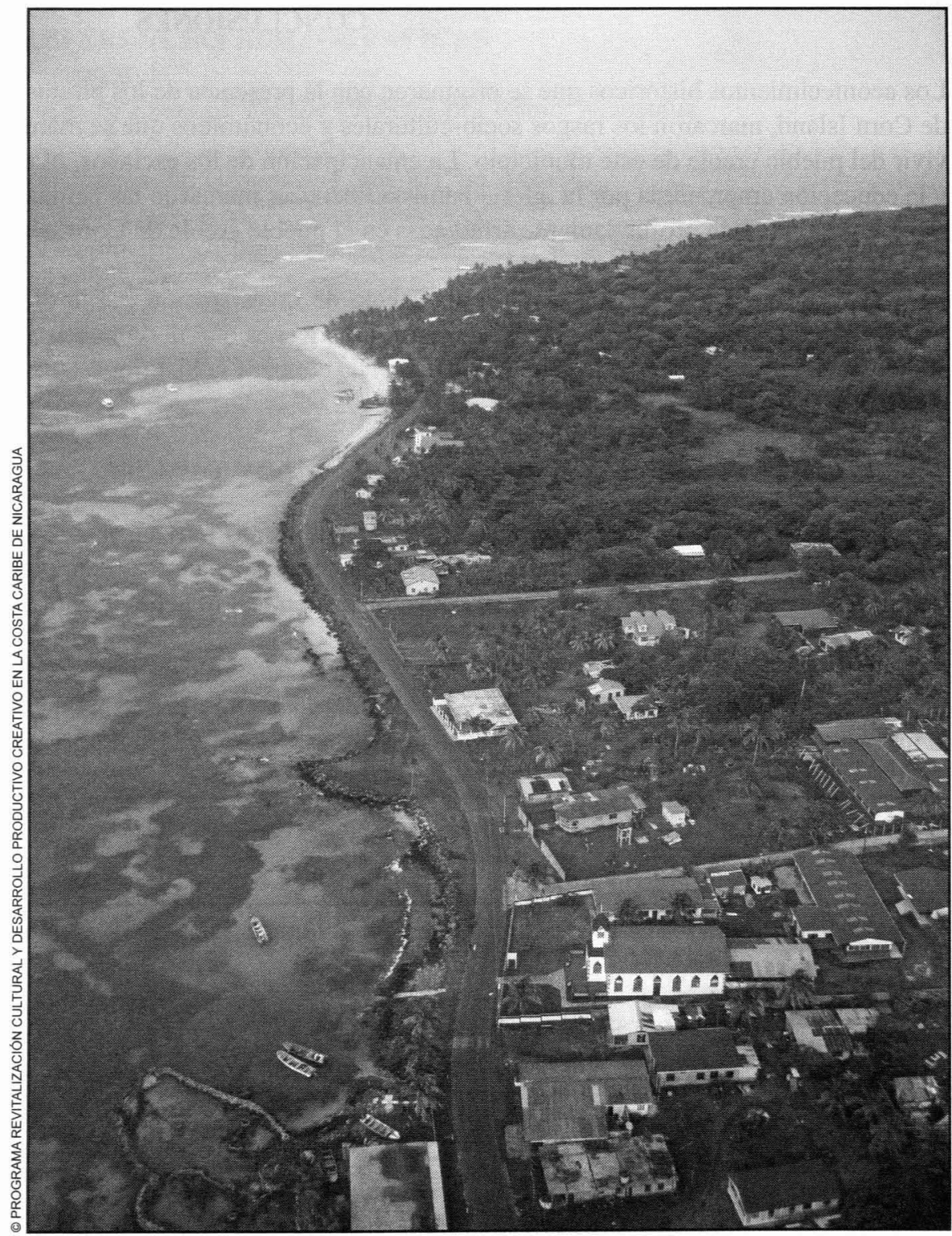

Vista aerea de la Ciudad. Corn Island 2011. Centro. Actualmente, el presidente de esta comisión es el Sr. Irod Ruiz, y sus integrantes lo conforman 22 miembros, entre hombres y mujeres de los diferentes sectores y barrios de la localidad.

\section{Desastres naturales que marcaron a Corn Island}

El primer desastre natural que recuerdan los pobladores de la Isla ocurrió en 1906. Este huracán destruyó totalmente la vegetación de la Isla. Se podía observar totalmente la isla de punta a punta. Cuenta el reverendo Edward Kelly, en

su diario personal, que después del desastre recibieron una ayuda solidaria de las islas de San Andrés y Providencia. Fue dinero en efectivo el cual se compartió equitativamente entre cada familia de la Isla.

El otro desastre que marcó dolorosamente a la Isla fue el huracán Joan el 21 de octubre de 1988. Este huracán destruyó más del $80 \%$ de la vegetación de la isla, principalmente los arboles de coco y frutales, lo que repercutió en la economía del Municipio. 


\section{CONCLUSIONES}

Los acontecimientos históricos que se originaron con la presencia de los piratas, bucaneros y colonos europeos en la isla de Corn Island, marcaron los rasgos socio-culturales y económicos que se manifiestan en el comportamiento y modo de vivir del pueblo creole de este municipio. La emancipación de los esclavos, el establecimiento de la institución religiosa y la educación emprendida por la iglesia bautista Ebenezer marcaron las pautas y las peculiaridades de las costumbres y tradiciones que están profundamente arraigadas en el pueblo creole de Corn Island en la actualidad.

La mayor problemática encontrada en este trabajo de investigación fue la dificultad para tener acceso a documentos escritos de la historia de la Isla, por lo que muchos jóvenes aún desconocen sus raíces culturales. Esperamos que este aporte contribuya a que la población actual de la Isla conozca la historia de sus ancestros.

\section{RECOMENDACIONES}

Se recomienda que las instancias pertinentes de los gobiernos municipal y regional publiquen en diversos medios toda esta información documentada ya que las personas conocedoras de esta rica y maravillosa historia son de avanzada edad y se necesita preservar correctamente esta documentación histórica del legado de nuestros antepasados creoles de la isla de Corn Island.

Se recomienda que las autoridades regionales incorporen dentro del sistema de educación en el municipio de Corn Island la historia oral de su población creole, como una materia dentro de los programas de Educación Primaria y Secundaria.

\begin{tabular}{|l|l|l|c|}
\hline \multicolumn{1}{|c|}{ Nombres y apellidos } & \multicolumn{1}{c|}{ Apodo } & \multicolumn{1}{c|}{ Ocupación } & Edad \\
\hline Dora Elena Hodgson Bryan & Dora Mama & Ama de casa & 84 \\
\hline Lorna Adela Hodgson Bryan & Nana & Ama de casa & 88 \\
\hline Hurvey John Quinn Downs & & Fabricante aceite de coco & \\
\hline McNeil Bryan Campbell & Makey & Agricultor & 84 \\
\hline Rosilda Lampson Downs & Sil & Costurera & 90 \\
\hline Rincard Arthur Hodgson Taylor & Rinki & Auxiliar de enfermería & 66 \\
\hline Vance Quinn Downs & & Pescador, carpintero & 62 \\
\hline Abraham Forbes Downs & & Pescador, albañil & 65 \\
\hline Bonnilyn Gloria Tucker Quinn & & Costurera & 87 \\
\hline Ardean Quinn Wilson & & Agricultor, celador & 69 \\
\hline
\end{tabular}

Otros portadores culturales que brindaron su valiosa información para este trabajo de investigación

1. Irod Erlin Ruiz Lam

2. Evis Ruby May Bodden

3. Nuvia Downs Zamora

4. Lestel Downs

5. Julia Jackson

6. Allan Taylor 


\section{REFERENCIAS BIBLIOGRÁFICAS}

Canzoneri, A. (1977). The 125th. Anniversary of Ebenezer Baptist Church Corn Island, Nicaragua.

Conzemius E. (1929). Les ILes Corn du Nicaragua, La Géographie, vol. LII, pp. 346-362.

Cunningham, M. \& Mairena, D. (2009). Diagnóstico Sociocultural de la costa Caribe de Nicaragua. San José, C.R.: UNESCO.

Decreto No. 3584: reglamento a la Ley No. 28, Estatuto de la autonomía de las regiones de la Costa Atlántica de Nicaragua. (1987, 30 de octubre). La Gaceta, pp. 2833-2838.

Díaz C., J. H. (1992). Introducción a la historia de América. Méjico.

Gonzalez I., J. J. (1989). El desarrollo de la pesca de langosta en Corn Island. Canada: SIFC.

Morgan, R. (1996). Meet Corn Island.

Nicaragua. Ministerio del Ambiente y Recursos Naturales (MARENA) (1999). Plan de acción para el manejo de los recursos naturales del municipio de Corn Island. Managua: MARENA.

The American Heritage Spanish Dictionary. (1986). Boston, Houghton Mifflin Company.

Tweedy \& Maureen. (1953). This is Nicaragua. England: East Anglian Magazine.

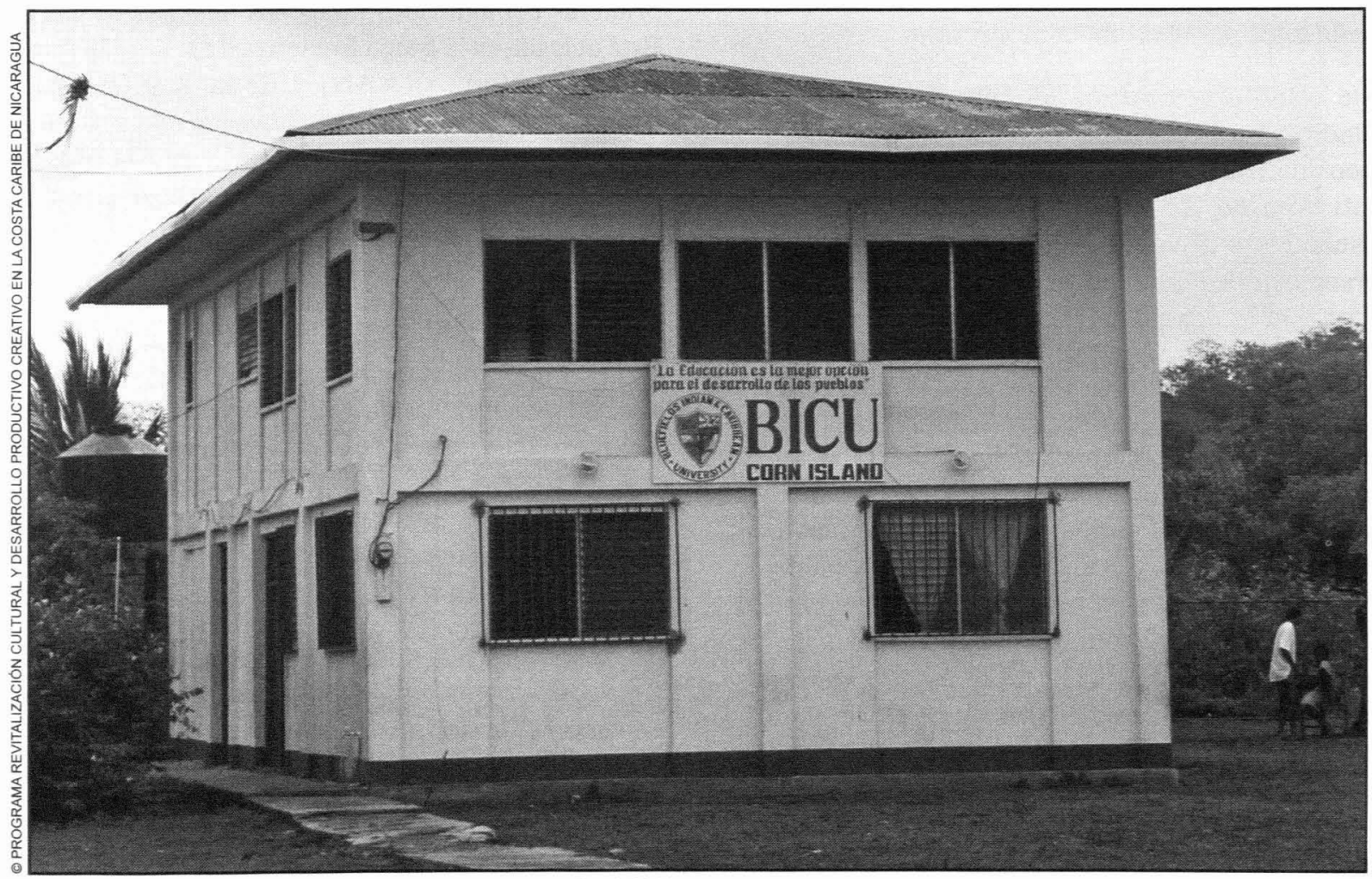

Bluefields Indian \& Caribbean University. Corn Island 2011. 